\title{
STABILITY THEOREMS FOR THE CONTINUOUS SPECTRUM OF A NEGATIVELY CURVED MANIFOLD \\ BY
}

HAROLD DONNELLY

\begin{abstract}
The spectrum of the Laplacian $\Delta$ for a simply connected complete negatively curved Riemannian manifold is studied. The Laplacian $\Delta_{0}$ of a simply connected constant curvature space $M_{0}$ is known up to unitary equivalence. Decay conditions are given, on the metric $g$ and curvature $K$ of $M$, which imply that the continuous part of $\Delta$ is unitarily equivalent to $\Delta_{0}$.
\end{abstract}

Introduction. Let $M$ be a complete simply connected Riemannian manifold having negative sectional curvatures. Since $M$ is complete, the Laplacian $\Delta$ of $M$ is a selfadjoint unbounded operator on $L^{2} M$. When $M$ is the symmetric space $M_{0}$ of constant negative curvature -1 , then $\Delta_{0}$ is known up to unitary equivalence, as summarized in $\S 2$. In particular, $\Delta_{0}$ has purely absolutely continuous spectrum.

The present paper is concerned with stability of the continuous part of $\Delta_{0}$ under perturbation of the metric $g_{0}$ on $M_{0}$. Theorem 4.12 gives decay conditions on the metric $g$ and curvature $K$ of $M$ which guarantee that $\Delta$ has the same absolutely continuous part as $\Delta_{0}$. Much weaker decay conditions on $K$ alone guarantee that $\Delta$ has no singular continuous spectrum, as specified in Theorem 5.7. Combining these results, one obtains criteria which ensure that $\Delta$ has the same continuous part as $\Delta_{0}$.

In our earlier paper [7], we established the above results for compactly supported perturbations of the metric on $\boldsymbol{M}_{\mathbf{0}}$. As well as obtaining stronger results, the current paper provides a better method, which may have other applications. One technique used essentially is to transplant the heat kernel and resolvent kernel from $M_{0}$ to $M$, as functions of the geodesic distance.

For background material on symmetric spaces and functional analysis, the reader may consult [7] and the references given there.

1. Heat kernels for complete Riemannian manifolds. The classical construction of a fundamental solution for the heat equation, as given in [1, pp. 204-215], uses repeatedly the hypothesis that one is working on a compact Riemannian manifold. However, as observed in [4, pp. 7-8] and [5, pp. 6-9], the usual method generalizes to any complete Riemannian manifold $M$ having bounded geometry. Here we say that $M$ has bounded geometry if its injectivity radius $\delta$ is bounded below and $\left\|\nabla^{i} R\right\|_{\infty} \leqslant D_{i}$, where $\nabla^{i} R$ is the $i$ th covariant derivative of the curvature tensor $R$ of $M$. In this section, we will show that the condition of bounded geometry can be

Received by the editors November 8, 1979 and, in revised form, December 2, 1979 and March 18, 1980.

AMS (MOS) subject classifications (1970). Primary 53C99, 58G99.

(C) 1981 American Mathematical Society 0002-9947/81/0000-0160/\$05.50 
weakened to assume only that the Ricci curvature and injectivity radius of $M$ are bounded below.

Let $\Delta$ be the Laplacian for $M$. Since $M$ is complete, the Laplacian $\Delta$ is an unbounded selfadjoint operator on $L^{2} M$. Therefore, the fundamental solution for the heat equation $\exp (-t \Delta) ; L^{2} M \rightarrow L^{2} M$ is well defined by Hilbert space theory. We say that $M$ has a good fundamental solution if $\exp (-t \Delta)$ is represented by a kernel $E(t, x, y)$ satisfying the properties:

P1. $\left(\partial / \partial t+\Delta_{2}\right) E(t, x, y)=0$ where $\Delta_{2}$ is the Laplacian acting in the second variable.

P2. $\lim _{t \rightarrow 0} E(t, x, y)=\delta(x, y)$, the Dirac delta measure.

P3. For $T>0$ arbitrary and $0 \leqslant t \leqslant T$, one has when $M$ is of dimension $n$ :

$$
|E(t, x, y)| \leqslant C_{1} t^{-n / 2} \exp \left(-C_{2} r^{2}(x, y) / t\right)
$$

where $C_{1}, C_{2}$ may depend only on $T$. Here $r$ is the geodesic distance from $x$ to $y$.

One has

THEOREM 1.1. Let $M$ be a complete negatively curved Riemannian manifold and $\Delta$ the Laplacian of $M$. Suppose that the Ricci curvature and injectivity radius of $M$ are bounded below. Then the heat equation problem on $M$,

$$
(\partial / \partial t+\Delta) f(x, t)=0, \quad f(x, 0)=f_{0}(x)
$$

has a good fundamental solution $E(t, x, y)$ satisfying properties $\mathrm{P} 1-\mathrm{P} 3$ above.

Furthermore $E(t, x, y)$ is unique and satisfies:

(Symmetry)

$$
E(t, x, y)=E(t, y, x)
$$

(Semigroup Property)

$$
E(t+s, x, y)=\int_{M} E(t, x, z) E(s, z, y) d z
$$

Proof. Let $\varepsilon<\delta$, where $\delta$ is the injectivity radius of $M$. Choose $\phi: R \rightarrow R$ to be a smooth function satisfying $\phi(\alpha)=1$ if $|\alpha|<\varepsilon / 2$ and $\phi(\alpha)=0$ if $|\alpha|>\varepsilon$. If $r$ is the geodesic distance between points in $M$, then set $\eta(x, y)=\phi(r(x, y))$.

A first approximation for $E$ is given by

$$
E_{1}(t, x, y)=(4 \pi t)^{-d / 2} \exp \left(-r^{2} / 4 t\right) \eta(x, y) .
$$

For $\eta=1$, this is just the fundamental solution for the heat equation on Euclidean space.

The proof requires some estimates concerning $E_{1}$ and related kernels:

Lemma 1.2. Denote $R_{1}=\left(\partial / \partial t+\Delta_{x}\right) E_{1}$. Then one has the estimate

$$
\left|R_{1}(t, x, y)\right| \leqslant C_{3} t^{-d / 2-1 / 2} \exp \left(-C_{4} r^{2} / t\right)
$$

for $0<t \leqslant T$, where $C_{3}, C_{4}$ may depend only on $T$.

Proof. If $r(x, y)<\delta$, let $\theta(x, y)$ denote the volume element in spherical polar coordinates centered at $x$. Thus if $f \in C_{0}^{\infty}\left(B_{\delta}(x)\right)$, where $B_{\delta}(x)$ is the ball of radius 
$\delta$ about $x$, one has

$$
\int_{M} f=\int f(r, \omega) \theta(r, \omega) d r d \omega
$$

where $(r, \omega)$ are spherical polar coordinates centered at $x$.

When $f(r)$ is a function depending only on the geodesic distance from $x$ to $y$, there is the well-known formula [15, p. 240]:

$$
\Delta f=\frac{-d^{2} f}{d r^{2}}-\frac{\theta^{\prime}}{\theta} \frac{d f}{d r}
$$

with $\theta^{\prime}=\partial \theta / \partial r$. Applying (1.3) and computing gives

$$
\begin{aligned}
R_{1}= & (4 \pi t)^{-d / 2} \exp \left(-r^{2} / 4 t\right) \\
& \cdot\left[\eta\left(\frac{r}{2 t}\right) \frac{\theta^{\prime}}{\theta}+\left(\frac{r}{t}\right) \eta^{\prime}(r)+\Delta \eta+\frac{1}{2} t^{-1}(1-d) \eta\right] .
\end{aligned}
$$

Since the Ricci curvature of $M$ is bounded below and $r<\delta$, in the support of $\eta$, a standard comparison theorem [3, p. 253] gives $\left|\theta^{\prime} / \theta+(1-d) / r\right|<B_{1}$. Note that $\theta^{\prime} / \theta+(1-d) / r$ is exactly the logarithmic derivative in $r$ of the Jacobian of the exponential map at $x$. Since $\eta$ is the constant for $r<\varepsilon / 2$, the same comparison theorem gives $|\Delta \eta| \leqslant B_{2}$. Thus

$$
\left|R_{1}\right| \leqslant B_{3} t^{-d / 2-1} r \exp \left(-B_{4} r^{2} / t\right)
$$

So

$$
\left|R_{1}\right| \leqslant B_{3} t^{-d / 2-1 / 2}\left[\frac{r^{2}}{t} \exp \left(\frac{-B_{4} r^{2}}{2 t}\right)\right]^{1 / 2} \exp \left(\frac{-B_{4} r^{2}}{2 t}\right) .
$$

The lemma now follows from the elementary inequality $\alpha e^{-k \alpha}<(k e)^{-1}$ applied to the quantity in brackets.

We may define

$$
A * B(t, x, y)=\int_{0}^{t} d s \int_{M} A(s, x, z) B(t-s, z, y) d z
$$

whenever the integrals converge absolutely. In this section only, one of the kernels $A, B$ will be compactly supported in $z$ for fixed $x, y$. Thus convergence of the integral over $M$ is no problem. To show convergence of the $t$ integral will require more careful estimates.

Denote $R_{i}=R_{1} * R_{1} * \cdots * R_{1}$ to be the $i$-fold convolution. Then we may write:

Lemma 1.4. For suitable constants $C_{5}, C_{6}$ one has

$$
\left|R_{i}(t, x, y)\right| \leqslant C_{5} t^{(d / 2+1 / 2)(i-2)} \exp \left(-C_{6} r^{2} / t\right)
$$

uniformly for $i \leqslant I, t \leqslant T$.

Moreover, for fixed $x, R_{i}(t, x, y)$ has $y$ support in $B_{i e}(x)$.

Proof. Since $R_{1}(t, x, y)=0$ if $r(x, y) \geqslant \varepsilon$, the definition of $R_{i}$ shows that $R_{i}(t, x, y)=0$ when $r(x, y) \geqslant i \varepsilon$. 
Lemma 1.2 gives the desired estimate for $R_{1}$. Suppose, by induction, that we have shown

$$
\left|R_{i-1}(t, x, y)\right| \leqslant B_{5} t^{(d / 2+1 / 2)(i-3)} \exp \left(-B_{6} r^{2} / t\right) .
$$

Now, for $i \geqslant 2$,

$$
R_{i}=R_{1} * R_{i-1}=\int_{0}^{t} d s \int_{M} R_{1}(s, x, z) R_{i-1}(t-s, z, y) d z
$$

So

$$
\begin{aligned}
\left|R_{i}(t, x, y)\right| \leqslant & \int_{0}^{t} d s C_{3} B_{5} s^{-d / 2-1 / 2}(t-s)^{(d / 2+1 / 2)(i-3)} \\
& \cdot \int_{\substack{r(x, z)<\varepsilon \\
r(y, z)<(i-1) \varepsilon}} \exp \left(\frac{-C_{4} r^{2}(x, z)}{s}\right) \exp \left(\frac{-B_{6} r^{2}(y, z)}{t-s}\right) d z .
\end{aligned}
$$

Using the estimate,

$$
r^{2}(x, y) / t \leqslant r^{2}(x, z) / s+r^{2}(y, z) /(t-s),
$$

which follows from the triangle inequality, we may write

$$
\left|R_{i}(t, x, y)\right| \leqslant B_{7} \int_{0}^{t} s^{-1 / 2}(t-s)^{-1 / 2+(d / 2+1 / 2)(i-2)} d s \exp \left(-B_{8} r^{2}(x, y) / t\right) .
$$

Setting $s=t \lambda$, we find that

$$
\begin{aligned}
\left|R_{i}(t, x, y)\right| \leqslant & B_{7} \exp \left(-B_{8} r^{2}(x, y) / t\right) t^{(d / 2+1 / 2)(i-2)} \\
& \cdot \int_{0}^{1} \lambda^{-1 / 2}(1-\lambda)^{-1 / 2+(d / 2+1 / 2)(i-2)} d \lambda .
\end{aligned}
$$

The $\lambda$ integral converges, so the lemma is established by induction.

For $i \geqslant 2$, the estimate of Lemma 1.4 shows that $R_{i}(t, x, y)$ extends continuously to $[0, \infty) \times M \times M$. Thus a convolution removes the singularity at $t=0$ of $R_{1}$. One may now use the arguments of [5] to obtain the fundamental solution $E$ on $M$.

Denote $S_{l j}=R_{2 l+j}$ for $l \geqslant 0$ and $3 \leqslant j \leqslant 4$. Then one has

Lemma 1.5. For suitable constants $C_{7}, C_{8}, C_{9}$, independent of $l, j$, we have for $0 \leqslant t \leqslant T$ :

$$
\left|S_{l, j}(t, x, y)\right| \leqslant \frac{C_{7} C_{8}^{l}}{l !} t^{(d / 2+1 / 2)(j-2)+l} \exp \left(\frac{-C_{9} r^{2}(x, y)}{t}\right) .
$$

Proof. Lemma 1.4 gives the result for $l=0, j=3,4$. We proceed by induction on $l$ :

$$
S_{l, j}=S_{l-1, j} * R_{2}
$$

So from Lemma 1.4 and the induction hypothesis,

$$
\begin{aligned}
\left|S_{l j}(t, x, y)\right| \leqslant & \int_{0}^{t} \frac{C_{7} C_{8}^{l-1}}{(l-1) !} s^{(d / 2+1 / 2)(j-2)+l-1} d s \\
& \cdot C_{5} \int_{\substack{d(z, y)<2 \varepsilon \\
d(z, x)<(2 l+j-2) \varepsilon}} \exp \left(\frac{-C_{9} r^{2}(x, z)}{s}\right) \exp \left(\frac{-C_{6} r^{2}(y, z)}{(t-s)}\right) d s .
\end{aligned}
$$


Here we may suppose that $C_{9}<C_{6} / 2$. Using the elementary inequality $d^{2}(x, y) / t$ $\leqslant d^{2}(x, z) / s+d^{2}(z, y) /(t-s)$ :

$$
\begin{aligned}
\left|S_{l, j}(t, x, y)\right| \leqslant & \int_{0}^{t} s^{(d / 2+1 / 2)(j-2)+l-1} d s \\
& \cdot \int C_{7} \frac{C_{8}^{l-1}}{(l-1) !} C_{5} \exp \left(\frac{-C_{6} r^{2}(y, z)}{2 T}\right) d z \exp \left(\frac{-C_{9} r^{2}(x, y)}{t}\right) .
\end{aligned}
$$

Since the Ricci curvature is bounded below, the volume element grows at most exponentially, $\theta(y, z) \leqslant \exp \left(B_{8} r(y, z)\right)[3$, p. 253]. Thus, the $z$ integral is bounded.

So

$$
\left|S_{l, j}(t, x, y)\right| \leqslant t^{(d / 2+1 / 2)(j-2)+l} C_{7} \frac{C_{8}^{l-1}}{l !} C_{5} B_{9} \exp \left(\frac{-C_{9} r^{2}(x, y)}{t}\right) .
$$

This yields the estimate required by the lemma:

$$
\left|S_{l, j}(t, x, y)\right| \leqslant \frac{C_{7} C_{8}^{l}}{l !} t^{(d / 2+1 / 2)(j-2)+l} \exp \left(\frac{-C_{9} r^{2}(x, y)}{t}\right) .
$$

Now denote $Q=\sum_{i=1}^{\infty}(-1)^{i} R_{i}$. By Lemma 1.5 , the series converges absolutely and one has

$$
|Q(t, x, y)| \leqslant C_{10} t^{-d / 2-1 / 2} \exp \left(\frac{C_{9} r^{2}(x, y)}{t}\right)
$$

when $0 \leqslant t \leqslant T$.

As in [1] and [5], a fundamental solution is obtained by setting $E=E_{1}-$ $E_{1} * Q$. The uniqueness, semigroup, and symmetry properties of $E$ follow, as in [5, p. 9], from Duhamel's principle.

This completes the proof of Theorem 1.1.

A crude estimate on the behavior of the heat kernel for large $t$ is given by

Corollary 1.6. Let $M$ be as in Theorem 1.1. Then the heat kernel $E(t, x, y)$ satisfies the estimate

$$
|E(t, x, y)| \leqslant A_{1} e^{A_{2} t} t^{-n / 2} \exp \left(-A_{3} r^{2}(x, y) / t\right)
$$

for some $A_{1}, A_{2}, A_{3}>0$.

Proof. Theorem 1.1 and property P3 give the required estimate for $t \leqslant T$ and any $T>0$. It suffices to show that, for large $t$, one has

$$
|E(t, x, y)| \leqslant A_{1} e^{A_{4} t} \exp \left(-A_{3} r^{2}(x, y) / t\right) .
$$

By property P3 we may write

$$
|E(1, x, y)| \leqslant C_{1} \exp \left(-C_{2} r^{2}(x, y)\right) .
$$

Assume by induction that (1.7) holds for $t \leqslant T$ and some $A_{3} \leqslant C_{2} / 2, T>2$. Let $T<t \leqslant T+1$.

The semigroup property reads

$$
E(t+1, x, y)=\int E(t, x, z) E(1, z, y) d z
$$


So

$$
|E(t+1, x, y)| \leqslant C_{1} A_{1} e^{A_{4} t} \int \exp \left(\frac{-A_{3} r^{2}(x, z)}{t}\right) \exp \left(-C_{2} r^{2}(z, y)\right) d z .
$$

Since $r^{2}(x, y) /(t+1) \leqslant r^{2}(x, z) / t+r^{2}(z, y) / 2$, we have

$$
|E(t+1, x, y)| \leqslant C_{1} A_{1} e^{A_{4} t} \int \exp \left(\frac{-C_{2} r^{2}(z, y)}{2}\right) d z \exp \left(\frac{-A_{3} r^{2}(x, y)}{t+1}\right) .
$$

Thus

$$
|E(t+1, x, y)| \leqslant A_{1} e^{A_{4}(t+1)} \exp \left(-A_{3} r^{2}(x, y) /(t+1)\right) .
$$

This completes the induction and proof of the corollary.

2. The constant curvature case. Let $M$ be a complete simply connected Riemannian manifold having constant curvature -1 . The Laplacian $\Delta$ of $M$ is identified, up to unitary equivalence, by the theory of special functions on $M$. These constant curvature spaces will be used as models in the present paper.

If $M$ is of dimension $n$, then the Laplacian $\Delta$ has purely absolutely continuous spectrum supported on the half line $\left[(n-1)^{2} / 4, \infty\right)$. Let $L^{2}\left(R^{+}, d x, \Re\right)$ denote the space of square Lebesgue integral $\Re$-valued functions on the positive real line. Here $\mathscr{N}$ is a Hilbert space of countable infinite dimension. It is well known [11, pp. 109, 131] that $\Delta$ is unitarily equivalent to the multiplication operator $f(x) \rightarrow$ $\left[(n-1)^{2} / 4+x^{2}\right] f(x)$, for $f \in L^{2}\left(R^{+}, d x\right.$, श $)$.

The spherical transform of Harish-Chandra [11] may be employed to obtain formulas representing the heat kernel and resolvent kernel of $\Delta$. However, for our purposes, a more elementary approach will suffice.

According to Theorem 1.1, $M$ has a good heat kernel $E(t, x, y)$. In fact, the Hadamard Cartan Theorem [3, p. 184] implies that $M$ has infinite injectivity radius. Furthermore, $M$ is a symmetric space and therefore admits a transitive group of isometries $G$. Uniqueness of the heat kernel gives $E(t, g x, g y)=E(t, x, y)$. Moreover, since $M$ has rank one, the isotropy group at each $x \in M$ is transitive on the unit sphere in $T_{x} M$, so $E(t, x, y)=E(t, r(x, y))$. Here $r(x, y)$ is the geodesic distance from $x$ to $y$. For background on symmetric spaces, the reader may consult [10].

The resolvent equation $(\Delta-z) f=0$ has a fundamental solution $R(z, x, y)$, analogous to the heat kernel. In fact, if $z$ lies in some left half-plane, $\operatorname{Re} z<-A_{2}$, then by Corollary 1.6, we may write

$$
R(z, x, y)=\int_{0}^{\infty} e^{t z} K(t, x, y) d t
$$

When $x \neq y,(2.1)$ expresses the resolvent kernel as the Laplace transform of the heat kernel. In particular, the kernel $R(z, x, y)$ exists for $\operatorname{Re} z<-A_{2}$ and $R(z, x, y)$ $=R(z, r(x, y))$.

Suppose $\operatorname{Re} z<-A_{2}$. Then since $R$ is a function of $r$ alone we have [15, p. 240]

$$
\Delta R=\frac{-d^{2} R}{d r^{2}}-\frac{\theta^{\prime}}{\theta} \frac{d R}{d r}
$$


In the constant curvature case [3, p. 253], $\theta=(\sinh r)^{n-1}$, so

$$
(\Delta-z) R=\frac{-d^{2} R}{d r^{2}}-(n-1) \operatorname{coth} r \frac{d R}{d r}-z R=0
$$

by definition of the resolvent $(\Delta-z)^{-1}$. Setting $x=\cosh r,(2.2)$ becomes

$$
\left(x^{2}-1\right) \frac{d^{2} R}{d x^{2}}+n x \frac{d R}{d x}+z R=0 .
$$

Denote $p$ to be the solution of $z=(n-1)^{2} / 4+p^{2}$ with $p$ having positive imaginary part for $\operatorname{Re} z<-A_{2}$. Set $m=n / 2-1$. Then the general solution of the ordinary differential equation (2.3) is of the form

$$
R(z, x)=\left(x^{2}-1\right)^{-m / 2}\left[a_{1}(z) P_{-1 / 2+\sqrt{-1} p}^{m}(x)+a_{2}(z) Q_{-1 / 2+\sqrt{-1} p}^{m}(x)\right]
$$

where $P, Q$ are the usual Legendre functions [17, I, pp. 65-67], for $x>1$.

Since $R$ represents the resolvent, for $\operatorname{Re} z<-A_{2}$, the coefficients $a_{1}, a_{2}$ are determined. In fact $R(z, x)$ must have the following properties: (i) $R(z, x)$ induces a bounded map $L^{2} M \rightarrow L^{2} M$, (ii) $R(z, x)$ has the same local singularity at $r=0$ as the Euclidean Green's function. Using (i), (ii) and the standard asymptotic formulas for Legendre functions [17, II, pp. 14, 15, 75, 221, 222], one obtains explicit formulas representing $a_{1}, a_{2}$. The actual expressions are rather cumbersome. Our main point is that (2.4) provides a continuation of the kernel $R(z, x)$ from $\operatorname{Re} z<-A_{2}$ to the $z$-plane, with a branch cut along the interval $\left[(n-1)^{2} / 4, \infty\right)$. The branch cut comes from extracting the square root $p=\left[z-(n-1)^{2} / 4\right]^{1 / 2}$.

The special function theory also shows that the analytically continued kernel $R(z, r)$ induces a bounded map $L^{2} M \rightarrow L^{2} M$ for $z \in\left[(n-1)^{2} / 4, \infty\right)$. Thus, by the uniqueness of analytic continuation, $R(z, r)$ must represent the resolvent $(\Delta-z)^{-1}$ for $z \in C-\operatorname{Spec} \Delta$.

3. Transplanted heat kernels. Let $M$ be a complete simply connected $n$-dimensional Riemannian manifold having negative sectional curvatures. By a theorem of Hadamard and Cartan [3, p. 183], the exponential map exp: $T_{p} M \rightarrow M$ is a diffeomorphism for each $p \in M$. Consequently, there is a system of spherical polar coordinates $(r, \omega)$ about $p$, with volume element $\theta(r, \omega)$. If $M_{0}$ is the simply connected complete space having constant curvature -1 , then $\theta_{0}=(\sinh r)^{n-1}$, independent of $p, \omega$.

Suppose that the metric on $M$ is obtained by perturbing the metric of $M_{0}$. We would like to give decay conditions on the metric $g$ and curvature $K$ of $M$ which guarantee that the Laplacian $\Delta$ of $M$ has the same absolutely continuous part as the Laplacian $\Delta_{0}$ of $M_{0}$. This section provides a technical device for attacking the problem of stability for the absolutely continuous spectrum. The main idea is to transplant the heat kernel $E_{0}$ from $M_{0}$ to $M$ by regarding $E_{0}(t, r)$ as a function of the geodesic distance $r$ on $M$.

Let $E_{0}(t)$ be the heat kernel of $M_{0}$ for fixed $t>0$. Recall from $\S 2$, that $E_{0}(t)$ depends only upon the geodesic $r_{0}$ between points in $M_{0}$. Consequently, we may define $F(t, x, y)=E_{0}(t, r(x, y))$, where $r(x, y)$ is the geodesic distance in $M$. 
Property P3 of $\S 1$ gives the estimate $|F(t, x, y)| \leqslant C_{1} \exp \left(-C_{2} r^{2}(x, y)\right)$ for fixed $t>0$.

By using the exponential maps at $p$, we may identify the differentiable manifolds underlying $M, M_{0}$. Suppose that, modulo this identification, the metric $g$ satisfies the decay conditions

$$
(1+\beta)^{-2} g_{0}(V, V) \leqslant g(V, V) \leqslant(1+\beta)^{2} g_{0}(V, V)
$$

for $V \in T_{x} M$. Here $\beta(x)=D_{1} \exp \left(-D_{2} r(x, p)\right)$, with $D_{2}>0$. For convenience, denote $\gamma(x)=r(x, p)$. Using (3.1), we see that $\left|\theta(p, x) / \theta_{0}(\gamma(x))\right|$ is bounded above and below by positive constants. This allows one to identify $L^{2} M$ and $L^{2} M_{0}$, via geodesic spherical coordinates about $p$. Moreover, the kernel $F$ induces bounded selfadjoint operators $F_{0}(t): L^{2} M_{0} \rightarrow L^{2} M_{0}$ and $F(t): L^{2} M \rightarrow L^{2} M$, which are unitarily equivalent.

We first observe

Lemma 3.2. Suppose that in (3.1), $\beta(\gamma)=D_{3} \exp \left(-D_{4} \gamma\right)$ with $D_{4}>n-1$. Then $F_{0}(t)-E_{0}(t)$ is Hilbert-Schmidt.

Proof. Let $r_{0}$ denote the geodesic distance in $M_{0}$. Then $r_{0}(p, x)=r(p, x)=$ $\gamma(x)$. The difference $P(t)=F_{0}(t)-E_{0}(t)$ has kernel $P(t, x, y)=E_{0}(t, r(x, y))-$ $E_{0}\left(t, r_{0}(x, y)\right)$.

Choose $\varepsilon<1$ so that $\varepsilon D_{4}>n-1$. Then if $r(x, y) \leqslant(1-\varepsilon) \gamma(x)$, the triangle inequality yields $\gamma(y)>\varepsilon \gamma(x)$. Consequently, $[1+\beta(\varepsilon \gamma)]^{-1} r_{0} \leqslant r<[1+\beta(\varepsilon \gamma)] r_{0}$, where $\gamma=\gamma(x)$.

Clearly

$$
|P(t, x, y)| \leqslant \int_{r_{0}}^{r}\left|\frac{\partial}{\partial r} E_{0}\right| d r .
$$

However, for fixed $t$, it is well known [5, pp. 6-9] that $\left|\partial E_{0} / \partial r\right|<$ $C_{3} \exp \left(-C_{4} r^{2}\right)$. So

$$
|P(t, x, y)| \leqslant C_{3} \exp \left(-C_{5} r_{0}^{2}(x, y)\right)\left(r-r_{0}\right) .
$$

Thus

$$
|P(t, x, y)| \leqslant C_{6} \beta(\varepsilon \gamma(x)) \exp \left(-C_{5} r_{0}^{2} / 2\right) .
$$

By applying the triangle inequality, we deduce that

$$
|P(t, x, y)| \leqslant C_{7} \beta(\varepsilon \gamma(x) / 2) \beta(\varepsilon \gamma(y) / 2) \exp \left(-C_{5} r_{0}^{2}(x, y) / 4\right)
$$

if $r(x, y) \leqslant(1-\varepsilon) \gamma(x)$. By symmetry, one has (3.3) when $r(x, y)<(1-\varepsilon) \gamma(y)$.

Now suppose that $r(x, y) \geqslant \max ((1-\varepsilon) \gamma(x),(1-\varepsilon) \gamma(y))$. Then using $|P(t, x, y)| \leqslant|F(t, x, y)|+\left|E_{0}(t, x, y)\right|$ we see that

$$
|P(t, x, y)| \leqslant C_{8} \beta(\varepsilon \gamma(x)) \beta(\varepsilon \gamma(y)) .
$$

Using (3.3), (3.4) and the condition $\varepsilon D_{4}>n-1$, we find

$$
\int_{M_{0} \times M_{0}}[P(t, x, y)]^{2} d x d y<\infty .
$$

So $P(t)$ is Hilbert-Schmidt. 
Now let

$$
G_{0}(2 t, x, y)=\int_{M_{0}} F(t, x, z) F(t, z, y) d z,
$$

so that $G_{0}(2 t)=F_{0}(t) \circ F_{0}(t)$, the composition. Of course, $G_{0}(2 t): L^{2} M_{0} \rightarrow L^{2} M_{0}$ is a bounded selfadjoint operator. Moreover, we have

Proposition 3.5. Suppose that in (3.1), $\beta=D_{3} \exp \left(-D_{4} \gamma(x)\right)$ with $D_{4}>n-1$. Then $E_{0}(2 t)-G_{0}(2 t)$ is trace class.

Proof. If $\varepsilon<1$, so that $\varepsilon D_{4}>n-1$, let $\mathfrak{N}$ be the operator of multiplication by $\exp \left(\varepsilon D_{4} \gamma(x) / 2\right)$. Employing the factorization trick of $[13$, p. 1190] we write

$$
\begin{aligned}
E_{0}(2 t)-G_{0}(2 t)= & {\left[E_{0}(t) \Re^{-1}\right]\left[\Re\left(E_{0}(t)-F_{0}(t)\right)\right] } \\
& +\left[\left(E_{0}(t)-F_{0}(t)\right) \Re\right]\left[\Re^{-1} F_{0}(t)\right],
\end{aligned}
$$

using the semigroup property of $E_{0}(t)$. As in Lemma 3.2, the inequalities (3.3) and (3.4) imply that each operator in brackets is Hilbert-Schmidt. So $E_{0}-G_{0}$ is a trace class.

The main result of this section is

THEOREM 3.6. Let $M$ be a complete simply connected negatively curved manifold whose metric is obtained by perturbing the metric $g_{0}$ of the constant curvature space $M_{0}$. Suppose that the metric $g$ of $M$ satisfies the decay condition (3.1) with $D_{4}>$ $n-1$.

Denote by $F(t): L^{2} M \rightarrow L^{2} M$ the selfadjoint operator obtained by transplanting $E_{0}(t)$ via $F(t, x, y)=E_{0}(t, r(x, y))$, where $r$ is the geodesic distance on $M$. Then, for any $t>0$, the absolutely continuous part of $F(t): L^{2} M \rightarrow L^{2} M$ is unitarily equivalent to $E_{0}(t): L^{2} M_{0} \rightarrow L^{2} M_{0}$.

Proof. We have observed that $F(t)$ is unitarily equivalent to $F_{0}(t): L^{2} M_{0} \rightarrow$ $L^{2} M_{0}$. By Proposition 3.5, $E_{0}(2 t)=E_{0}(t) \circ E_{0}(t)$ has $G_{0}(2 t)=F_{0}(t) \circ F_{0}(t)$ as a trace class perturbation. Thus $E_{0}(2 t)$ and $G_{0}(2 t)$ have the same absolutely continuous part by a theorem of Birman and Kato [2, p. 98]. Theorem 3.6 now follows by extracting the positive square roots $E_{0}(t), F_{0}(t)$ of $E_{0}(2 t), F_{0}(2 t)$.

4. The absolutely continuous spectrum. Let us continue in the framework of $\S 3$. We have shown that the operator $F(t): L^{2} M \rightarrow L^{2} M$ with kernel $F(t, x, y)=$ $E_{0}(t, r(x, y))$ has absolutely continuous part which is unitarily equivalent to $\exp \left(-t \Delta_{0}\right): L^{2} M_{0} \rightarrow L^{2} M_{0}$. In the present section, the kernel $F$ will be employed as a parametrix to construct the fundamental solution $E(t, x, y)$ of the heat equation on $M$. Curvature decay conditions will be given which guarantee that $E(t)$ and $F(t)$ have the same absolutely continuous part.

In preparation, some technical lemmas are required:

Lemma 4.1. Let $\lambda_{1} \geqslant \lambda_{2}>0$. Then

(i) $\sup _{s>0}\left(\lambda_{1} \operatorname{coth} \lambda_{1} s-\lambda_{2} \operatorname{coth} \lambda_{2} s\right)=\lambda_{1}-\lambda_{2}$;

(ii) $\sup _{s>0}\left(\lambda_{1} \operatorname{coth} \lambda_{1} s-1 / s\right)=\lambda_{1}$. 
Proof. Calculus.

Let $p \in M$ and suppose that $\gamma(x)=r(x, p)$ is the geodesic distance of $x$ from $p$. Denote by $K(x, \pi)$ the sectional curvature of the two-plane $\pi$ at $x$. Then one has

LEMma 4.2. Assume that for all $(x, \pi)$, we have $|K(x, \pi)+1|<C_{1} \exp \left(-C_{2} \gamma(x)\right)$, for $C_{2}>0$. Then, for $r(x, y)<(1-\varepsilon) \gamma(x), 0<\varepsilon<1$, we may write

$$
\left|\left(\theta^{\prime} / \theta\right)(x, y)-\left(\theta_{0}^{\prime} / \theta_{0}\right)(r(x, y))\right|<D_{3} \exp \left(-\varepsilon C_{2} \gamma(x)\right)
$$

where $\theta^{\prime}$ is the partial derivative with respect to $r(x, y)$.

Proof. By the triangle inequality, $\gamma(y)>\varepsilon \gamma(x)$. For simplicity, let us abbreviate $\gamma=\gamma(x)$ and $h(\gamma)=C_{1} \exp \left(-C_{2} \gamma\right)$.

If $h(\varepsilon \gamma)<\frac{1}{2}$, then by a standard comparison theorem [3, p. 284]:

$$
\begin{aligned}
& \left|\left(\theta^{\prime} / \theta\right)(x, y)-\left(\theta_{0}^{\prime} / \theta_{0}\right)(r(x, y))\right| \\
& \leqslant(n-1) \sup _{s<(1-\varepsilon) \gamma} \mid \operatorname{coth}(\sqrt{1+h(\varepsilon \gamma)} s) \sqrt{1+h(\varepsilon \gamma)} \\
& \quad-\operatorname{coth}(\sqrt{1-h(\varepsilon \gamma)} s) \sqrt{1-h(\varepsilon \gamma)} \mid .
\end{aligned}
$$

So, by Lemma 4.1,

$$
\begin{aligned}
\left|\left(\theta^{\prime} / \theta\right)(x, y)-\left(\theta_{0}^{\prime} / \theta_{0}\right)(r(x, y))\right| & \leqslant(n-1)|\sqrt{1+h(\varepsilon \gamma)}-\sqrt{1-h(\varepsilon \gamma)}| \\
& \leqslant B_{1} h(\varepsilon \gamma) .
\end{aligned}
$$

On the other hand, if $h(\varepsilon \gamma) \geqslant \frac{1}{2}$, then by [3, p. 284] and Lemma 4.1,

$$
\begin{aligned}
\mid\left(\theta^{\prime} / \theta\right)(x, y)- & \left(\theta_{0}^{\prime} / \theta_{0}\right)(r(x, y)) \mid \\
& \leqslant \sup _{s<(1-\varepsilon) \gamma}|\sqrt{1+h(\varepsilon \gamma)} \operatorname{coth}(\sqrt{1+h(\varepsilon \gamma)} s)-1 / s| \\
& \leqslant(n-1) \sqrt{1+h(\varepsilon \gamma)} \leqslant B_{2} h(\varepsilon \gamma)
\end{aligned}
$$

for $h(\varepsilon \gamma) \geqslant \frac{1}{2}$. This proves Lemma 4.2.

We may now state

THEOREM 4.3. Let $M$ be a complete simply connected $n$-dimensional Riemannian manifold having negative sectional curvatures. Fix $p \in M$, and suppose that for all $(x, \pi)$, one has $|K(x, \pi)+1| \leqslant C_{1} \exp \left(-C_{2} \gamma(x)\right)$. Here $\gamma(x)$ is the geodesic distance of $x$ from $p$.

If $C_{2}>n-1$, then the operators $\exp (-t \Delta)$ and $F(t): L^{2} M \rightarrow L^{2} M$ have unitarily equivalent absolutely continuous part.

Proof. We imitate the constructions of $\S 1$, using $F$ as a parametrix to obtain a representation of the heat kernel $E$ of $M$. Several lemmas are required. As observed in $[6$, p. 840$]$, the curvature decay condition guarantees that $\left|\theta(p, x) / \theta_{0}(\gamma(x))\right|$ is bounded above and below by positive constants. Choose $0<\varepsilon<1$ so that $\varepsilon C_{2}>n$ -1 . Then one has

Lemma 4.4. Denote $R_{1}=\left(\partial / \partial t+\Delta_{x}\right) F(t, x, y)$. Then for $0<t<T$ :

$$
\left|R_{1}(t, x, y)\right| \leqslant B_{3} \exp \left(-C_{2}[\gamma(x)+\gamma(y)] \varepsilon / 2\right) \exp \left(-B_{4} r^{2}(x, y) / 2 t\right) t^{-n / 2-1 / 2}
$$

where $B_{3}, B_{4}$ depend only upon $T$. 
Proof. One has

$$
\left(\frac{\partial}{\partial t}+\Delta\right) F(t, x, y)=\left[\frac{-\theta^{\prime}}{\theta}(x, y)+\frac{\theta_{0}^{\prime}}{\theta_{0}}(r(x, y))\right] \frac{\partial}{\partial r} E_{0}(t, r) .
$$

Now it is well known [5, pp. 6-9] that for $0<t \leqslant T$ :

$$
\left|\partial E_{0}(t, r) / \partial r\right| \leqslant D_{4} t^{-n / 2-1 / 2} \exp \left(-B_{4} r^{2}(x, y) / t\right) .
$$

If $r(x, y) \leqslant(1-\varepsilon) \gamma(x)$, then by Lemma 4.2 :

$$
\left|R_{1}(t, x, y)\right| \leqslant D_{3} \exp \left(-C_{2} \varepsilon \gamma(x)\right) D_{4} t^{-n / 2-1 / 2} \exp \left(-B_{4} r^{2}(x, y) / t\right) .
$$

By the triangle inequality:

$$
\left|R_{1}(t, x, y)\right| \leqslant \exp \left(-C_{2}[\gamma(x)+\gamma(y)] \varepsilon / 2\right) \exp \left(-B_{4} r^{2}(x, y) / 2 t\right) D_{5} t^{-n / 2-1 / 2} \text {. }
$$

Similarly, Lemma 4.4 follows if $r(x, y)<(1-\varepsilon) \gamma(y)$.

Now suppose $r(x, y) \geqslant(1-\varepsilon) \gamma(x)$ and $r(x, y) \geqslant(1-\varepsilon) \gamma(y)$. Since the curvature of $M$ is bounded below [3, p. 284],

$$
\left|\left(\theta^{\prime} / \theta\right)(x, y)-\left(\theta_{0}^{\prime} / \theta_{0}\right)\right|<B_{5}
$$

So

$$
\begin{aligned}
\left|R_{1}(t, x, y)\right| \leqslant & D_{4} B_{5} t^{-n / 2-1 / 2} \exp \left(-B_{4} r^{2}(x, y) / 2 t\right) \\
& \cdot \exp \left(-B_{4}\left[\gamma^{2}(x)+\gamma^{2}(y)\right](1-\varepsilon)^{2} / 4 t\right),
\end{aligned}
$$

which establishes Lemma 4.4, when $r(x, y) \geqslant \max (\gamma(x), \gamma(y))(1-\varepsilon)$.

As in the proof of Theorem 1.1, we denote $R_{i}=R_{1} * R_{1} * \cdots * R_{1}$ to be the $i$-fold convolution.

We have

LEMMA 4.5. For suitable constants $B_{6}, B_{7}$, one has

$$
\left|R_{i}(t, x, y)\right| \leqslant B_{6} t^{(d / 2+1 / 2)(i-2)} \exp \left(-B_{7} r^{2}(x, y) / t\right) \exp \left(-C_{2} \varepsilon[\gamma(x)+\gamma(y)] / 2\right)
$$

uniformly if $i \leqslant I, t \leqslant T$.

Proof. The proof is analogous to that of Lemma 1.4.

Define $S_{l, j}=R_{2 l+j}$ for $l \geqslant 0$; derive

LEMMA 4.6. For suitable constants $B_{8}, B_{9}, B_{10}$, independent of $l, j$, one has

$$
\begin{aligned}
\left|S_{l, j}(t, x, y)\right| \leqslant & \left(B_{8} B_{9}^{l} / l !\right) t^{(d / 2+1 / 2)(j-2)+l} \\
& \cdot \exp \left(-C_{2} \varepsilon[\gamma(x)+\gamma(y)] / 2\right) \exp \left(-B_{10} r^{2}(x, y) / t\right) .
\end{aligned}
$$

Proof. The proof is similar to the proof of Lemma 1.5.

If $Q=\sum_{i=0}^{\infty}(-1)^{i} R_{i}$, then the series converges absolutely and

$$
|Q(t, x, y)| \leqslant B_{11} t^{-d / 2-1 / 2} \exp \left(-B_{12} r^{2}(x, y) / t\right) \exp \left(-C_{2} \varepsilon[\gamma(x)+\gamma(y)] / 2\right)
$$

when $0 \leqslant t \leqslant T$. Moreover, one has the estimate

$$
\begin{aligned}
|F * Q(t, x, y)| \leqslant & A_{1} t^{1 / 2} \exp \left(-A_{2} r^{2}(x, y) / t\right) \\
& \cdot \exp \left(-C_{2} \varepsilon[\gamma(x)+\gamma(y)] / 2\right) .
\end{aligned}
$$

As in the proof of Theorem 1.1, we find that

$$
E=F-F * Q \text {. }
$$


It is now not difficult to show that $E-F$ defines a Hilbert-Schmidt operator. We have in fact,

LEMMA 4.9. For any $t>0$, the kernel

$$
P(t, x, y)=\exp \left(C_{2} \varepsilon \gamma(x) / 2\right)[E(t, x, y)-F(t, x, y)]
$$

defines a Hilbert-Schmidt operator.

Proof. Using (4.7) and (4.8) we find that

$$
\begin{aligned}
& \int_{M \times M}[P(t, x, y)]^{2} d x d y \\
& \quad \leqslant A_{1}^{2} t \int \exp \left(-2 A_{2} r^{2}(x, y) / t\right) \exp \left(-\varepsilon C_{2} \gamma(y)\right) d x d y<\infty
\end{aligned}
$$

since $\varepsilon C_{2}>n-1$.

Let $G=F^{2}$ be the composition

$$
G(2 t, x, y)=\int_{M} F(t, x, z) F(t, z, y) d z .
$$

Then one has the estimate

$$
|G(t, x, y)| \leqslant A_{3} t^{-n / 2} \exp \left(-A_{4} r^{2}(x, y) / t\right)
$$

for $0<t \leqslant T$. In general, $G(t) \neq F(t)$, since the measure on $M$ is different from that of $M_{0}$, so the semigroup property of $\exp \left(-t \Delta_{0}\right)$ is lost. However, $G(t)$ is still unitarily equivalent to $\exp \left(-t \Delta_{0}\right)$.

We may state

Lemma 4.10. For any $t>0$, the kernel $E(2 t, x, y)-G(2 t, x, y)$ defines a trace class operator.

Proof. As in [13, p. 1190] we exploit the semigroup property of $E$ :

$$
\begin{aligned}
E(2 t)-G(2 t)=[ & \left.E(t) \Re^{-1}\right][\Re(E(t)-F(t))] \\
& +[(E(t)-F(t)) \Re]\left[\Re^{-1} F(t)\right]
\end{aligned}
$$

where $\Re$ is the multiplication operator $f(x) \rightarrow \exp \left(C_{2} \varepsilon \gamma(x) / 2\right) f(x)$. A computation, similar to the proof of Lemma 4.9, shows that each operator in brackets is Hilbert-Schmidt.

Lemma 4.10 and the theorem of Birman and Kato [2, p. 98] imply that $E(2 t)$ and $G(2 t)$ have unitarily equivalent absolutely continuous part. Theorem 4.3 now follows by extracting the unique positive square roots $E(t)$ and $F(t)$ of $E(2 t)$ and $G(2 t)$.

REMARK 4.11. It would be logically flawless to omit $\$ 1$ and to construct the heat kernel $E$ on $M$ directly from $F$ as parametrix. However, such a presentation might be slightly misleading. The more general Theorem 1.1 also seems to have independent interest.

We may now collect the results of $\$ \S 3$ and 4 to state our main result on the absolutely continuous spectrum of $\Delta$ : 
THEOREM 4.12. Let $M$ be a complete simply connected Riemannian manifold having negative sectional curvatures. Suppose that the metric of $M$ is obtained by perturbation from the standard metric $g_{0}$ on the simply connected space of constant curvature -1 .

If $g, K$ denote the metric and curvature of $M$, then we impose the decay conditions:

(i) $(1+\beta)^{-2} g_{0}(V, V) \leqslant g(V, V) \leqslant(1+\beta)^{2} g_{0}(V, V)$ for $V \in T_{x} M$, and

(ii) $|K(x, \pi)+1| \leqslant h$ for $\pi$ a two plane at $x \in M$.

Here $h(x)=C_{1} \exp \left(-C_{2} \gamma(x)\right)$ and $\beta(x)=C_{3} \exp \left(-C_{2} \gamma(x)\right)$. Moreover, $\gamma(x)=$ $r(x, p)$ is the geodesic distance of $x$ from a fixed $p \in M$. We assume that $C_{2}>n-$ 1 , where $n$ is the dimension of $M$.

Under these conditions the absolutely continuous part of the Laplacian $\Delta: L^{2} M \rightarrow$ $L^{2} M$ is unitarily equivalent to $\Delta_{0}: L^{2} M_{0} \rightarrow L^{2} M_{0}$.

Proof. By Theorem 3.6 and condition (i), the operators $F(t): L^{2} M \rightarrow L^{2} M$ and $\exp \left(-t \Delta_{0}\right): L^{2} M_{0} \rightarrow L^{2} M_{0}$ have the same absolutely continuous part. However, $F(t)$ has the same absolutely continuous part as $\exp (-t \Delta)$ by condition (ii) and Theorem 4.3. Since $\Delta_{0}$ is purely absolutely continuous, Theorem 4.12 follows.

REMARK 4.13. Theorem 4.12 is a considerable improvement over the corresponding result in the author's earlier paper [7, p. 3]. It was shown there that $\exp (-t \Delta)-$ $\exp \left(-t \Delta_{0}\right)$ is trace class if the metric on $M$ is obtained by a compactly supported perturbation of the metric on $M_{0}$. The method used there requires control over the higher order derivatives of the metric, while conditions (i) and (ii) only restrict the metric $g$ and curvature $K$.

5. Singular continuous spectrum. Let $M$ be a complete simply connected Riemannian manifold having negative sectional curvatures. In this section we give decay conditions, on the curvature $K$ of $M$, which guarantee that the associated Laplacian $\Delta$ has no singular continuous spectrum. By the limiting absorption principle, it suffices to show that the resolvent $R(x)=(\Delta-z)^{-1}$ has good upper and lower boundary values $R^{+}(z), R^{-}(z)$ on the real axis. Actually, we will extend $R(z)$ across the real axis, except for a countable set of values which may cluster only at $(n-1)^{2} / 4$.

If $M_{0}$ is the simply connected complete space having constant curvature -1 , then the special functions results of $\S 2$ allow us to continue the resolvent, $R_{0}(z)=$ $\left(\Delta_{0}-z\right)^{-1}$. In fact, fix a point $\alpha \in\left((n-1)^{2} / 4, \infty\right)$ and a sufficiently small relatively compact open neighborhood $U_{\alpha}$ of $\alpha$. Since $\alpha \in \operatorname{Spec}\left(\Delta_{0}\right), R_{0}(z)$ cannot be continued from the upper half-plane to $U_{\alpha}$ as an operator $L^{2} M_{0} \rightarrow L^{2} M_{0}$. However, let us introduce the weighted spaces

$$
L^{2, s}\left(M_{0}\right)=\left\{\left.f(x)\left|\int_{M_{0}}\right| f(x)\right|^{2} e^{25 \gamma(x)} d x<\infty\right\}
$$

where $\gamma(x)=r(x, p)$, the geodesic distance from a fixed $p \in M_{0}$. Of course, for $s>0, L^{2, s} \subset L^{2} \subset L^{2,-s}$.

One has

Lemma 5.1. Let $s>0$ and $\alpha \in\left((n-1)^{2} / 4, \infty\right)$ be given. Then the resolvent $R_{0}(z)$ extends from the upper half-plane to a neighborhood $U_{\alpha}$ of $\alpha$, as a bounded operator $R_{0}^{+}(z): L^{2, s}\left(M_{0}\right) \rightarrow L^{2,-s}\left(M_{0}\right)$. 
Proof. In $\S 2$, we obtained a kernel $R_{0}(z, x, y)$ depending only on $z$ and the geodesic distance $r(x, y)$. The kernel represented $R_{0}(z)$ for $z \in C-$ $\left[(n-1)^{2} / 4, \infty\right)$. Moreover, $R_{0}(z, x, y)$ extended to the whole $z$ plane with a branch cut along $\left[(n-1)^{2} / 4, \infty\right)$.

Choose a smooth function $\chi(x, y)=\chi(r(x, y))$, with $\chi(r)=1$ for $r<\frac{1}{2}$, and $\chi(r)=0$ for $r>1$. We may write $R_{0}(z, x, y)=\chi(x, y) R_{0}+(1-\chi) R_{0}$. Although $\chi(x, y) R_{0}$ has a singularity on the diagonal, it follows from standard properties of pseudodifferential operators that $\chi(x, y) R_{0}$ defines a bounded operator $L^{2} M_{0} \rightarrow$ $L^{2} M_{0}$ [12, pp. 110-112]. Therefore $\chi R_{0}$ certainly extends to $U_{\alpha}$ as a bounded operator $L^{2, s}\left(M_{0}\right) \rightarrow L^{2,-s}\left(M_{0}\right)$.

The more interesting part of the proof involves $R_{1}(z, x, y)=(1-\chi) R_{0}(z, x, y)$. From (2.4) and the ensuing discussion, we have the estimate

$$
R_{1}(z, x, y)=O\left(\left|e^{[-(n-1) / 2+\sqrt{-1} p] r(x, y)}\right|\right)
$$

where $z=(n-1)^{2} / 4+p^{2}$ and $p>0$ for $z>(n-1)^{2} / 4$.

Denote

$$
R_{2}(z, x, y)=\exp (-s \gamma(x)) R_{1}(z, x, y) \exp (-s \gamma(y)) .
$$

Then $R_{2}$ is the kernel associated to $R_{1}$ via the natural identification $\exp (s \gamma(x))$ : $L^{2, s} \rightarrow L^{2}$. It suffices to show that $R_{2}$ extends as a bounded operator $L^{2} \rightarrow L^{2}$. However, by the triangle inequality,

$$
R_{2}(z, x, y)=O\left(e^{-[(n-1) / 2] r(x, y)-[\gamma(x)+\gamma(y)] s / 2}\right)
$$

for $z \in U_{\alpha}$, and $U_{\alpha}$ sufficiently small.

Now fix $p \in M_{0}$ and choose geodesic spherical coordinates $(r, \omega)$ about $p$. In these coordinates, the measure $d x=(\sinh r)^{n-1} d r d \omega$. Denote

$$
R_{3}(z, x, y)=\left(\gamma^{-1} \sinh \gamma(x)\right)^{(n-1) / 2} R_{2}(z, x, y)\left(\gamma^{-1} \sinh \gamma(y)\right)^{-(n-1) / 2}
$$

to be the operator associated to $R_{2}$ through the natural map

$$
\left(\gamma^{-1} \sinh \gamma\right)^{(n-1) / 2}: L^{2}\left(M_{0}, d x\right) \rightarrow L^{2}\left(T_{p} M_{0}, r^{n-1} d r d \omega\right) .
$$

Then, by the triangle inequality,

$$
R_{3}(z, x, y)=O\left(e^{-[\gamma(x)+\gamma(y)] s / 2}\right)
$$

It suffices to show that $R_{3}$ extends to $U_{\alpha}$ as a map on $L^{2}\left(T_{p} M_{0}, r^{n-1} d r d \omega\right)$. However, the kernel $R_{3}$ is Hilbert-Schmidt, so it actually defines a compact operator.

We now transplant the kernel $R_{0}(z, x, y)$ from $M_{0}$ to $M$ and define $S(z, x, y)=$ $R_{0}(z, r(x, y))$, where $r(x, y)$ is the geodesic distance on $M$. Denote

$$
L^{2, s}(M)=\left\{\left.f(x)\left|\int_{M}\right| f(x)\right|^{2} e^{2 s \gamma(x)} d x<\infty\right\}
$$

where $d x$ is the natural measure of $M$.

Suppose that the curvature $K$ of $M$ satisfies the decay condition

$$
|K(x, \omega)+1| \leqslant h(x)
$$


for $\omega$ a two-plane in $T_{x} M$. We denote $h(x)=C_{1} \exp \left(-C_{2} \gamma(x)\right)$ for $C_{2}>0$. Then, as observed in [6, pp. 8-10], the ratio $\left|\theta(r, \omega) / \theta_{0}(r)\right|$ of volume elements in spherical normal coordinates is bounded above and below by positive constants. Then the proof of Lemma 5.1 shows that $S(z, x, y)$ extends across the real axis to define an operator $S^{+}(z): L^{2, s}(M) \rightarrow L^{2,-s}(M), s>0$.

It will be important to study the operator with kernel

$$
Q(z, x, y)=\left(\frac{-\theta_{0}^{\prime}}{\theta_{0}}(r(x, y))+\frac{\theta^{\prime}}{\theta}(x, y)\right) \frac{\partial}{\partial r} S(z, r(x, y)) .
$$

Recall that $z=(n-1)^{2} / 4+p^{2}$ with $\sqrt{-1} p<0$ for $z<(n-1)^{2} / 4$.

We have

LEMMA 5.4. Suppose that the curvature $K$ satisfies the decay conditions (5.2) with $C_{2}>0$. Let $0<s<\min \left(n-1, C_{2}\right) / 2$. Then

(i) The kernel $Q(z, x, y)$ defines a compact operator $L^{2, s}(M) \rightarrow L^{2, s}(M)$ for $z \in C$ $-\left[(n-1)^{2} / 4, \infty\right)$.

(ii) Given $\alpha \in\left((n-1)^{2} / 4, \infty\right)$, the operator $Q(z)$ extends from the upper half $z$ plane to a neighborhood $U_{\alpha}$ of $\alpha$, as a compact operator $Q^{+}(z): L^{2, s}(M) \rightarrow L^{2, s}(M)$.

Proof. Let $P(z, x, y)=\overline{Q(z, y, x)}$. The kernel $P$ is the formal adjoint of $Q$ on $C_{0}^{\infty}(M)$. Since compactness is preserved under taking adjoints, it suffices to show that $P$ defines a compact operator $L^{2,-s}(M) \rightarrow L^{2,-s}(M)$.

Denote

$$
P_{1}(z, x, y)=\exp (-s \gamma(x)) P(z, x, y) \exp (s \gamma(y)) \text {. }
$$

Since compactness is preserved under composition with bounded operators, we need only show that $P_{1}: L^{2} M \rightarrow L^{2} M$ defines a compact operator.

Define $\chi(r)$ to be a smooth function satisfying $\chi(r)=1$ for $r<\frac{1}{2}$ and $\chi(r)=0$ for $r>1$. Denote $\chi(x, y)=\chi(r(x, y))$. Then we may write $P_{1}=P_{2}+P_{3}$ where $P_{2}=\chi P_{1}$ and $P_{3}=(1-\chi) P_{1}$.

According to Lemma 4.2, the quantity

$$
\chi(x, y)\left|\left(\theta^{\prime} / \theta\right)(x, y)-\left(\theta_{0}^{\prime} / \theta_{0}\right)(r(x, y))\right| e^{-s \gamma(x)} e^{s \gamma(y)}
$$

is bounded and approaches zero for $\gamma(x)$ or $\gamma(y)$ large. Here we employ the condition $s<C_{2} / 2$. Using a standard lemma on pseudodifferential operators [12, pp. 110-112] and the definition (5.3) of $Q$, we see that $P_{2}: L^{2} M \rightarrow L^{2} M$ is compact. This follows essentially from Rellich's lemma.

Now consider $P_{3}(z, x, y)$. For $r(x, y) \geqslant \frac{1}{2}$, one has the estimate

$$
|(\partial / \partial r) S(z, r(x, y))|=O\left(\left|e^{[-(n-1) / 2+\sqrt{-1} p] r(x, y)}\right|\right)
$$

which follows from (2.4) and standard asymptotic formulas involving Legendre functions [17, pp. 221-222].

If $z \in C-\left((n-1)^{2} / 4, \infty\right)$, then $\operatorname{Im}(p)>0$. Moreover, in Lemma 5.4(ii), if $U_{\alpha}$ is sufficiently small, one has $\operatorname{Im}(p)>-\delta$, for any $\delta>0$. Thus, we may assume that

$$
|(\partial / \partial r) S(z, r(x, y))|=O\left(e^{[-(n-1) / 2+\delta] r(x, y)}\right)
$$

where $\delta>0$ can be forced to be arbitrarily small. 
Let $h(x, y)$ be the characteristic function of the set $\{(x, y) \mid r(x, y)>$ $(1-\varepsilon) \gamma(x)\}$, where $0<\varepsilon<1$ will be specified later. One has $P_{3}=P_{4}+P_{5}$, with $P_{4}=(1-h) P_{3}$ and $P_{5}=h P_{3}$.

For $P_{4}$, one may use (5.3), (5.5), and Lemma 4.2 to yield the estimate

$$
\left|P_{4}(z, x, y)\right|=O\left(e^{-\left[s+\varepsilon C_{2}\right] \gamma(x)} e^{[-(n-1) / 2+\delta] r(x, y)} e^{s \gamma(y)}\right) .
$$

Introducing spherical coordinates $(r, \omega)$ about $p$, recall that $\left|\theta(r, \omega) /(\sinh r)^{n-1}\right|$ is bounded above and below by positive constants, as a consequence of our curvature decay conditions [6, pp. 8-10]. We may identify $L^{2}(M, d x)$ and $L^{2}\left(T_{p} M, r^{n-1} d r d \omega\right)$ via $f \rightarrow f\left[\theta(r, \omega) / r^{n-1}\right]^{1 / 2}$. Then the kernel $P_{6}: L^{2}\left(T_{p} M\right) \rightarrow$ $L^{2}\left(T_{p} M\right)$, associated to $P_{4}$, is of order

$$
\left|P_{6}(z, x, y)\right|=O\left(e^{\left[(n-1) / 2-s-e C_{2}\right] \gamma(x)} e^{[-(n-1) / 2+\delta] r(x, y)} e^{[-(n-1) / 2+s] \gamma(y)}\right) .
$$

Using the triangle inequality $\gamma(x) \leqslant r(x, y)+\gamma(y)$ yields

$$
\left|P_{6}(z, x, y)\right|=O\left(e^{-e C_{2} \gamma(x)} e^{(\delta-s) r(x, y)}\right) .
$$

If $\delta<s$ and $0<\mu<s-\delta$, then applying the triangle inequality $\gamma(y) \leqslant \gamma(x)+$ $r(x, y)$, one obtains

$$
\left|P_{6}(z, x, y)\right|=O\left(e^{\left(-\varepsilon C_{2}+\mu\right) \gamma(x)} e^{-\mu \gamma(y)}\right) .
$$

Choosing $\mu<\varepsilon C_{2}$, we see that $P_{6}: L^{2}\left(T_{p} M\right) \rightarrow L^{2}\left(T_{p} M\right)$ is Hilbert-Schmidt and consequently compact. Since compactness is preserved under composition with bounded operators, $P_{4}: L^{2} M \rightarrow L^{2} M$ is compact.

Finally, we must deal with $P_{5}=h P_{3}$. Now, the curvature of $M$ is bounded below and, thus [3, p. 284], $\left|\theta / \theta(x, y)-\theta_{0}^{\prime} / \theta_{0}(r(x, y))\right|$ is bounded on $\boldsymbol{M} \times \boldsymbol{M}$. Combining this observation with (5.2) and (5.5), one finds

$$
\left|P_{5}(z, x, y)\right|=O\left(h(x, y) e^{-s \gamma(x)} e^{[-(n-1) / 2+\delta] r(x, y)} e^{s \gamma(y)}\right) .
$$

Using the isomorphism between $L^{2}\left(T_{p} M\right)$ and $L^{2} M$ as above, we identify $P_{5}$ with an operator $P_{7}: L^{2}\left(T_{p} M\right) \rightarrow L^{2}\left(T_{p} M\right)$. The kernel $P_{7}$ satisfies

$$
\left|P_{7}(z, x, y)\right|=O\left(h(x, y) e^{[(n-1) / 2-s] \gamma(x)} e^{[-(n-1) / 2+\delta] r(x, y)} e^{[-(n-1) / 2+s] \gamma(y)}\right) .
$$

One may choose $\varepsilon, \delta$ sufficiently small so that

$$
(1-\varepsilon)((n-1) / 2-\delta)-((n-1) / 2-s)=\beta>0 .
$$

Recalling that $h(x, y)$ is the characteristic function of the set $\{(x, y) \mid r(x, y)>$ $(1-\varepsilon) \gamma(x)\}$, we find

$$
\left|P_{7}(z, x, y)\right|=O\left(e^{-\beta \gamma(x)} e^{[-(n-1) / 2+s] \gamma(y)}\right) .
$$

Then $P_{7}: L^{2}\left(T_{p} M\right) \rightarrow L^{2}\left(T_{p} M\right)$ is Hilbert-Schmidt. Consequently, $P_{5}$ is compact.

This completes the proof of Lemma 5.4.

We may now extend the resolvent kernel of $M$ :

Proposition 5.6. Let $0<s<\min \left(n-1, C_{2}\right) / 2$ be given. Suppose that $\alpha \in R-$ $\Lambda$, where $\Lambda$ will be some countable set of points which may cluster only at $(n-1)^{2} / 4$. Then the resolvent $R(z)=(\Delta-z)^{-1}$ extends from the upper half-plane to a neighborhood $U_{\alpha}$ of $\alpha$ as a bounded operator $R^{+}(z): L^{2, s} \rightarrow L^{2,-s}$. 
Proof. Fix $\alpha \in R-(n-1)^{2} / 4$ and $U_{\alpha}$ so that the conclusion of Lemma 5.4 is satisfied. One has the second resolvent equation

$$
S^{+}(z)=R(z)\left[I+Q^{+}(z)\right] \text {. }
$$

From Lemma 5.4, we see that $I+Q^{+}(z): L^{2, s} \rightarrow L^{2, s}$ is Fredholm. Using a standard lemma on families of compact operators [14, p. 370] we see that $\operatorname{ker}\left[I+Q^{+}(z)\right]=0$ except for finitely many $z \in \Lambda_{\alpha} \subset U_{\alpha}$. For $z \in U_{\alpha}-\Lambda_{\alpha}$, the Fredholm alternative allows us to continue $R(z)$ by

$$
R^{+}(z)=S^{+}(z)\left[I+Q^{+}(z)\right]^{-1} \text {. }
$$

Our main result for the present section is

THEOREM 5.7. Let $M$ be a complete simply connected negatively curved manifold. Suppose that $K(x, \pi)$ denotes the sectional curvature of the two-plane $\pi$ in $T_{x} M$. Impose the curvature decay condition $|K(x, \pi)+1| \leqslant C_{1} \exp \left(-C_{2} \gamma(x)\right)$, where $\gamma(x)$ $=r(x, p)$ is the geodesic distance from $p$ in $M$. Then the Laplacian $\Delta$ of $M$ has no singular continuous spectrum.

Proof. Proposition 5.6 shows that $R(z)$ has good lower boundary values $R^{+}(z)$ : $L^{2, s} \rightarrow L^{2,-s}$. Similarly, one shows that there are good upper boundary values $R^{-}(z): L^{2, s} \rightarrow L^{2,-s}$. Theorem 5.7 now follows from the limiting absorption principle [7, p. 64], [8, p. 1202].

REMARK 5.8. In the author's earlier paper [7], it was shown that if $\Delta$ is the Laplacian of a metric obtained by a compactly supported perturbation of the metric on the constant curvature space $M_{0}$, then $\Delta$ has no singular continuous spectrum. Theorem 5.7 is much stronger, since one need only satisfy curvature decay conditions. No restraints are imposed on the other derivatives of the metric $g$. In fact, $M$ need not be obtained from $M_{0}$ by a perturbation of $g_{0}$.

6. Identifying the Laplacian up to unitary equivalence. One may combine Theorems 4.12 and 5.7 to obtain a condition guaranteeing stability of the continuous spectrum:

THEOREM 6.1. Let $M$ be a complete simply connected Riemannian manifold having negative sectional curvatures. Suppose that the metric of $M$ is obtained by perturbation from the standard metric $g_{0}$ on the simply connected space of constant curvature -1 .

If $g, K$ denote the metric and curvature of $M$, then we impose the decay conditions:

(i) $(1+\beta)^{-2} g_{0}(V, V) \leqslant g(V, V) \leqslant(1+\beta)^{2} g_{0}(V, V)$ for $V \in T_{x} M$, and

(ii) $|K(x, \pi)+1| \leqslant h$ for $\pi$ a two-plane at $x \in M$.

Here $h(x)=C_{1} \exp \left(-C_{2} \gamma(x)\right)$ and $\beta(x)=C_{3} \exp \left(-C_{2} \gamma(x)\right)$. Moreover, $\gamma(x)=$ $r(x, p)$ is the geodesic distance of $x$ from a fixed $p \in M$. We assume that $C_{2}>n-$ 1 , where $n$ is the dimension of $M$.

Under these conditions the continuous part of the Laplacian $\Delta: L^{2} M \rightarrow L^{2} M$ is unitarily equivalent to $\Delta_{0}: L^{2} M_{0} \rightarrow L^{2} M_{0}$.

The eigenvalues $\lambda$ embedded in the continuum, that is $\lambda>(n-1)^{2} / 4$, were discussed in the author's earlier paper [6]. 
THEOREM 6.2. Suppose that conditions (i) and (ii) of Theorem 6.1 are satisfied, and also

(iii) $\int_{0}^{\infty}\left\|\nabla_{\omega} K\right\| e^{2 r} d r<D_{1}$,

(iv) $\int_{0}^{\infty}\left\|\nabla_{\omega}^{2} K\right\| e^{2 r} d r<D_{2}$

for some constants $D_{1}, D_{2}>0$. Here $\nabla_{\omega} K$ is the covariant derivative in geodesic spherical coordinates $(r, \omega)$ of any sectional curvature $K$ along the geodesics emanating from $p$.

Then $\Delta$ has the same continuous part as $\Delta_{0}$. Moreover, $\Delta$ has no eigenvalue $\lambda>(n-1)^{2} / 4$.

Finally, by adding one more condition, we obtain a stability theorem for the entire spectrum:

TheOREM 6.3. Let $M$ be as in Theorem 6.2 and assume that

(v) $K \leqslant-1$.

Then $\Delta$ is unitarily equivalent to $\Delta_{0}$.

Proof. If $K \leqslant-1$ then the spectrum of $\Delta$ is bounded below by $(n-1)^{2} / 4[16, \mathrm{I}$, p. 88], [18, p. 498]. Moreover, $(n-1)^{2} / 4$ cannot occur as an eigenvalue [6, p. 11], [16, II, p. 4] when $K \leqslant-1$.

\section{BIBLIOGRAPHY}

1. M. Berger, P. Gauduchon and E. Mazet, Le spectre d'une variété Riemannienne, Lecture Notes in Math., vol. 194, Springer-Verlag, Berlin and New York, 1971.

2. M. S. Birman, Existence conditions for wave operators, Izv. Akad. Nauk. SSSR Ser. Mat. 27 (1953), pp. 883-906; English transl., Amer. Math. Soc. Transl. (2) 54 (1966), 91-118.

3. R. Bishop and R. Crittenden, Geometry of manifolds, Academic Press, New York and London, 1964.

4. J. Cheeger and S. T. Yau, A lower bound for the heat kernel, (preprint).

5. H. Donnelly, Asymptotic expansions for the compact quotients of properly discontinuous group actions, Illinois J. Math. 23 (1979), 485-496.

6. ___ Eigenvalues embedded in the continuum for negatively curved manifolds, Michigan Math. J. (to appear).

7. Spectral geometry for certain noncompact Riemannian manifolds, Math. Z. 169 (1979), 63-76.

8. N. Dunford and J. T. Schwartz, Linear operators. Part II, Interscience, New York and London, 1963.

9. P. Hartman, Ordinary differential equations, Wiley, New York, 1964.

10. S. Helgason, Differential geometry and symmetric spaces, Academic Press, New York, 1962.

11. _ Harmonic analysis on homogeneous spaces, Proc. Sympos. Pure Math., vol. 26, Amer. Math. Soc., Providence, R. I., 1973, pp. 101-146.

12. L. Hormander, Fourier integral operators. I, Acta Math. 127 (1971), 79-183.

13. A. Jensen and T. Kato, Asymptotic behavior of the scattering phase for exterior domains, Comm. Partial Differential Equations 3 (1978), 1165-1195.

14. T. Kato, Perturbation theory for linear operators, Grundlehren der Math. Wissenschaften, vol. 32, Springer-Verlag, Berlin and New York, 1976.

15. V. K. Patodi, Curvature and the eigenforms of the Laplace operator, J. Differential Geometry 5 (1971), 233-249.

16. M. Pinsky, The spectrum of the Laplacian on a manifold of negative curvature. I, II, J. Differential Geometry 13 (1978), 87-91; J. Differential Geometry (to appear).

17. L. Robin, Fonctions sphériques de Legendre. Tome I, II, III, Gauthier-Villars, Paris, 1959.

18. S. T. Yau, Isoperimetric constants and the first eigenvalue of a complete Riemannian manifold, Ann. Sci. Ecole Norm. Sup. 8 (1975), 487-507.

Department of Mathematics, Purdue University, West Lafayette, Indiana 47907 